\title{
Reactive Amyloidosis and Familial Mediterranean Fever (FMF)
}

Key words: amyloid A, serum amyloid A, MEFV, amyloidogenesis

Amyloidosis is not the consequence of a single disease entity but a variety of different disease processes resulting in the extracellular deposition of insoluble fibrillar proteins in various organs (1).

The diagnosis is based on characteristic biopsy findings. Amyloid fibrils are stained with HE as non-structural eosinophilic substance and show orange-green birefringence under polarized light after Congo red staining. By electron microscopy, amyloid fibril appear similar to piano wire, measuring approximately 7.5 to $10 \mathrm{~nm}$ in diameter and forming rigid, nonbranching, linear aggregate fibrils.

Currently, many different types of amyloidosis are classified based on the precursor protein; systemic amyloidosis is classified under the four most common types, e.g., amyloid A (AA), amyloid light chain, amyloid transthyretin, and amyloid $\beta 2$ microglobulin. AA amyloidosis is a reactive systemic amyloidosis, associated with rheumatic disease, chronic inflammatory conditions including familial Mediterranean fever (FMF), chronic infections, and some malignant diseases. In the early 1980s, tuberculosis and bronchiectasia were the most important underlying conditions, but the importance of the diseases was reduced with the advent of effective anti-microbial chemotherapy (2). Recently, more frequently associated conditions are rheumatoid arthritis (RA) and other inflammatory diseases.

The deposition of AA amyloid involves multiple organ systems, usually without any symptoms. Approximately $25 \%$ of patients with amyloidosis prospectively present with renal involvement (3), the most common cause of death in these patients. The typical clinical presentation is proteinuria due to the deposition of amyloid fibrils in the glomerulus. Initially, the proteinuria may be mild and intermittent, but later sustained and increasing to the nephrotic stage, progressing to renal failure.

The current understanding of the processes involved in AA amyloidosis is that it is the result of a two-step mechanism (4). First, the production of AA amyloid requires the synthesis of circulating precursor proteins in sufficient quantities for deposition. In chronic and recurrent acute inflammatory conditions, macrophage activation leads to the synthesis and release of cytokines, such as interleukin 1 , interleukin 6 , and tumor necrosis factor $\alpha$. Mainly, the liver synthesizes serum amyloid A (SAA) proteins under the influence of above described cytokines. Atherosclerotic smooth muscle cells, endothelial cells, and macrophages also partly synthesize these proteins
(5). SAA protein is secreted and associated with the third fraction of high-density lipoprotein particles.

Second, the continuous maintenance of a high concentration of intact SAA or fragmented AA protein and conformational changes of these proteins may be necessary to generate a fibrillar condition in which preformed fibril (nucleus) will be formed, consequently it will be the rapid fibril extension of AA amyloid fibril as well as other amyloidosis (6).

As for the quality of SAA, humans have four types of SAA genes located on the short arm of chromosome 11. SAA1 and SAA2 are highly homologous and increase rapidly in reaction to inflammation. SAA3 is a pseudogene and SAA4 is constitutive, but does not respond to acute inflammation. In the Japanese population of adult patients with RA, the homozygosity for the SAA1 $\gamma$ allele is an important risk factor for AA amyloidosis (7). However, in Caucasian patients with juvenile chronic arthritis, the presence of the homozygous SAA $1 \alpha$ allele is a significant risk factor (8). These findings are controversial. Recently, a novel single-nucleotide polymorphism at the 5'flanking region of SAA1 was reported as a risk of AA amyloidosis. Thus, the discrepancy in the SAA allele between populations may be explained (9).

Although the SAA protein is an approximately $12.5 \mathrm{kDa}$ component, AA protein isolated from fibrils range between 3.5 and $7 \mathrm{kDa}(10)$. Proteolysis, which may be tissue specific, is responsible for this fragmentation. Neutrophil elastase, and monocyto-proteases and some cathepsins have been shown to be capable of digesting SAA. Fragmentation has also been implicated in amyloidogenesis (5).

FMF is an autosomal recessive disease and idiopathic febrile disorder characterized by attacks of fever accompanied by serositis (11). This disease affects certain ethnic groups, mainly Armenians, Sephardic Jewish, Turks, and Arabs. Reactive amyloidosis (renal amyloidosis) is the most serious manifestation (11), since it ultimately determines the patient's prognosis.

The gene (MEFV) causing FMF has been mapped to the short arm of chromosome 16, and cloned by the International Consortium in 1997 (12). MEFV responsible for FMF spans 10 exons, and encodes a 781 amino acid protein weighing 86 $\mathrm{kDa}$. The International Consortium called this protein pyrin. Although the precise physiological function of pyrin is not known, it has been suspected that pyrin is an inflammatory regulator of the neutrophil-mediated inflammatory response.

How does pyrin itself, encoding various mutations, concretely associate with the fibrillogenesis of amyloid? There are many possibilities. The first involves the factors facilitating hyperproduction of SAA, second is inhibiting the digestion of 
SAA by blocking various proteases, third is enhancement of amyloid enhancing factor, fourth is direct acceleration of fibril formation, and finally, inhibition of digestion of formed fibril. However the details of the mechanisms remain obscure.

More than 26 mutations in MEFV have been associated with FMF, and pairs of MEFV mutations can be identified. Five founder mutations, V726A, M694V, M694I, M680I, and E148Q account for $74 \%$ of FMF chromosomes. Most studies have indicated that the presence or homozygosity of M694V mutation is the main cause among patients who developed amyloidosis. However, some investigators have found that other mutations are also important for amyloidogenesis in FMF. Furthermore, polymorphisms in the SAA1 gene, to be exact, the SAA1 $\alpha / \alpha$ allele, significantly influence susceptibility to amyloidosis in FMF (13), as well as in Caucasian RA patients with reactive amyloidosis described above. Not only MEFV genes, but also other gene factors, such as the SAA1 genotype, or environmental factors (living area, ethnic differences, gender, use of colchicine) seem to play a role in the extent and severity of febrile attacks and the development to amyloidosis in FMF.

Several studies have clearly shown that treatment with colchicine for FMF can prevent febrile attacks, the development of renal amyloidosis, and while promote the recovery from nephrotic syndrome secondary to amyloidosis (14). This indicates that organ injury due to amyloid deposition is reversible, even if amyloid deposition does not disappear microscopically. According to Gillmore's recent report (15), AA amyloid deposits exist in a state of dynamic turnover, and the outcome of AA amyloidosis is favorable when a lower SAA concentration is maintained. In FMF, control of SAA using colchicine and other medications is also necessary to promote a better prognosis.

Although more than 4,000 patients with FMF have been reported worldwide, FMF patients in Japan are very rare. The report of Tomiyama et al (16) describes the first case associated with end stage renal disease due to renal amyloidosis, and diagnosed by analyzing the mutation in MEFV gene.

That report was very important and described an interesting case to consider the relation between FMF and AA amyloidogenesis.

\author{
Naoki Takahashi, MD, Eiichi Suzuki, MD \\ and Fumitake GEJYo, MD \\ The Department of Homeostatic Regulation and Development, \\ Graduate School of Medical and Dental Sciences, \\ Niigata University, 1-757 Asahimachi-dori, Niigata 951-8510
}

\section{References}

1) Glenner GG. Amyloid deposits and amyloidosis. The $\beta$-fibrilloses (first of two parts). N Engl J Med 302: 1283-1292, 1980.

2) Pras M. Familial Mediterranean fever: from the clinical syndrome to the cloning of the pyrin gene. Scand J Rheumatol 27: 92-97, 1998 (editorial).

3) Montoli A, Minola E, Stabile F, et al. End-stage renal failure from renal amyloidosis of the AA type associated with giant lymph node hyperplasia (Castleman's disease). Am J Nephrol 15: 142-146, 1995.

4) Kushner I, Rzewnicki DL. The acute phase response: general aspects. Baillieres Clin Rheumatol 8: 513-530, 1994.

5) Cunnane G. Amyloid precursors and amyloidosis in inflammatory arthritis. Curr Opin Rheumatol 13: 67-73, 2001.

6) Naiki H, Gejyo F. Kinetic analysis of amyloid fibril formation. Methods Enzymol 309: 305-318, 1999.

7) Baba S, Masago SA, Takahashi $T$, et al. A novel allelic variant of serum amyloid A, SAA1 $\gamma$ : genomic evidence, evolution, frequency, and implication as a risk factor for reactive systemic AA-amyloidosis. Hum Mol Genet 4: 1083-1087, 1995.

8) Booth DR, Booth SE, Gillmore JD, Hawkins PN, Pepys MB. SAA1 alleles as risk factors in reactive systemic AA amyloidosis. Amyloid 5: 262265, 1998.

9) Moriguchi M, Terai C, Kaneko H, et al. A novel single-nucleotide polymorphism at the 5'-flanking region of SAA1 associated with risk of type AA amyloidosis secondary to rheumatoid arthritis. Arthritis Rheum 44: 1266-1272, 2001.

10) Sipe JD. Amyloidosis. Annu Rev Biochem 61: 947-975, 1992.

11) Sohar E, Gafni J, Pras M, Heller H. Familial Mediterranean fever. A survey of 470 cases and review of the literature. Am J Med 43: 227-253, 1967.

12) The International FMF Consortium. Ancient missense mutations in a new member of the RoRet gene family are likely to cause familial Mediterranean fever. Cell 90: 797-807, 1997.

13) Cazeneuve $C$, Ajrapetyan $H$, Papin $S$, et al. Identification of MEFV-independent modifying genetic factors for familial Mediterranean fever. Am J Hum Genet 67: 1136-1143, 2000.

14) Zemer D, Livneh A, Langevitz P. Reversal of the nephrotic syndrome by colchicine in amyloidosis of familial Mediterranean fever. Ann Intern Med 116: 426, 1992 (letter).

15) Gillmore JD, Lovat LB, Persey MR, Pepys MB, Hawkins PN. Amyloid load and clinical outcome in AA amyloidosis in relation to circulating concentration of serum amyloid A protein. Lancet 358: 24-29, 2001.

16) Tomiyama N, Oshiro S, Higashiuesato $Y$, et al. End-stage renal disease associated with Familial Mediterranean fever. Intern Med 41: 221-224, 2002. 